\title{
Importância do fisioterapeuta no período gestacional
}

\author{
Importance of the physiotherapist in the gestational period \\ Importancia del fisioterapeuta en el periodo gestacional
}

Recebido: 28/08/2021 | Revisado: 04/09/2021 | Aceito: 04/09/2021 | Publicado: 06/09/2021

\author{
Josiany Resplandes da Silva \\ ORCID: https://orcid.org/0000-0001-9154-6997 \\ Instituto Educacional Santa Catarina, Brasil \\ E-mail: josianyresplandes@outlook.com \\ Weslane Lira Resplandes \\ ORCID: https://orcid.org/0000-0002-9187-4998 \\ Instituto Educacional Santa Catarina, Brasil \\ E-mail: weslanylira@outlook.com \\ Karla Camila Correia da Silva \\ ORCID: https://orcid.org/0000-0003-1538-7028 \\ Instituto Educacional Santa Catarina, Brasil \\ Faculdade Guaraí, Brasil \\ E-mail: karlacamilac@yahoo.com.br
}

\begin{abstract}
Resumo
A Gravidez é um processo fisiologico natural compreendido pela sequencia de adaptacoe ocorrida no corpo da mulher a parti da fertilizacao. A preparacao do corpo para uma gestacao envolve alguns ajustes fisiologicos, onde apresenta um grande aumento de hormios causando uma afroxidao ligamentar e mudancas mecanicas no esqueleto e biomecanicas. (Mantle e Polden, 2005). Em decorrência da gravidez é frequente o aparecimento de desconfortos, dores e alterações no sistema musculoesquelético. No decorrer da história as pessoas que tinham um estilo de vida mais ativo durante o período da gestação tiveram mais benefícios na hora do parto. Organismos nacionais e internacionais, e com os avanços tecnológicos e médicos, buscando proporcionar as gestantes segurança na gestação, incentivam elas a praticar exercícios físicos, visando a promoção da saúde, evitando a mortalidade materna e fetal. O fisioterapeuta é profissional adequado para atenuar as sintomatologias gestacional, trabalhando com medidas preventivas. O presente estudo fundamentou-se em uma pesquisa bibliográfica do tipo descritiva, que busca demonstrar a importância do fisioterapeuta durante o período gestacional e o período pós-parto. A prática de exercícios durante a gravidez é fundamental, pois melhora o condicionamento físico, ajuda no metabolismo, diminui edema, trabalha a respiração e condiciona o corpo para o trabalho de parto. Através da pesquisa realizada, nota-se que o fisioterapeuta tem ação importante tanto no período gestacional quanto no período de pós-parto, através de técnicas que melhoram a dor, a postura corporal, contribui no parto, minimizando lesões no assoalho pélvico.
\end{abstract}

Palavras-chave: Fisioterapia gestacional; Gestação; Atuação do fisioterapeuta.

\begin{abstract}
Pregnancy is a natural physiological process understood by the sequence of adaptation that takes place in the woman's body from fertilization. The preparation of the body for a pregnancy involves some physiological adjustments, where it presents a great increase of hormones causing a ligamentous loosening and mechanical changes in the skeleton and biomechanics. (Mantle and Polden, 2005). As a result of pregnancy, discomfort, pain and changes in the musculoskeletal system are frequent. Throughout history, people who had a more active lifestyle during the gestation period had more benefits at the time of childbirth. National and international organizations, and with technological and medical advances, seeking to provide pregnant women with safety during pregnancy, encourage them to practice physical exercise, aiming to promote health, avoiding maternal and fetal mortality. The physiotherapist is an adequate professional to alleviate gestational symptoms, working with preventive measures. The present study was based on a descriptive bibliographic research, which seeks to demonstrate the importance of the physiotherapist during the gestational period and the postpartum period. The practice of exercises during pregnancy is essential, as it improves physical conditioning, helps with metabolism, reduces edema, works on breathing and conditions the body for labor. Through the research carried out, it is noted that the physiotherapist plays an important role both in the gestational period and in the postpartum period, through techniques that improve pain, body posture, contribute to childbirth, minimizing injuries to the pelvic floor.
\end{abstract}

Keywords: Gestational physiotherapy; Gestation; Performance of the physiotherapist.

\section{Resumen}

El embarazo es un proceso fisiológico natural entendido por la secuencia de adaptación que tisne lugar en el cuerpo de la mujer a partir de la fecundación. La preparación del cuerpo para un embarazo implica algunos ajustes fisiológicos, 
donde presenta un gran aumento de hormonas provocando un aflojamiento de ligamentos y cambios mecánicos en el esqueleto y biomecánica. (Mantle y Polden, 2005). Como consecuencia del embarazo, son frecuentes las molestias, el dolor y los cambios en el sistema musculoesquelético. A lo largo de la historia, las personas que tuvieron un estilo de vida más activo durante el período de gestación tuvieron más beneficios en el momento del parto. Organismos nacionales e internacionales, y con avances tecnológicos y médicos, que buscan brindar seguridad a las mujeres embarazadas durante el embarazo, las incentivan a practicar ejercicio físico, con el objetivo de promover la salud, evitando la mortalidad materna y fetal. El fisioterapeuta es un profesional adecuado para aliviar los síntomas gestacionales, trabajando con medidas preventivas. El presente estudio se basó en una investigación bibliográfica descriptiva, que busca demostrar la importancia del fisioterapeuta durante el período gestacional y posparto. La práctica de ejercicios durante el embarazo es fundamental, ya que mejora el acondicionamiento físico, ayuda al metabolismo, reduce el edema, trabaja la respiración y acondiciona el organismo para el parto. A través de la investigación realizada, se observa que el fisioterapeuta juega un papel importante tanto en el período gestacional como en el posparto, mediante técnicas que mejoran el dolor, la postura corporal, contribuyen al parto, minimizando las lesiones en el piso pélvico.

Palabras clave: Fisioterapia gestacional; Gestación; Actuación del fisioterapeuta.

\section{Introdução}

Da fertilização ao nascimento do bebê, o corpo da mulher passa por diversas transformações e adaptações fisiológicas. Em decorrência da gravidez é frequente o aparecimento de desconfortos, dores e alterações no sistema musculoesquelético. No $1^{\circ}$ trimestre de gestação, as articulações e ligamentos tornam-se frouxos e instáveis, ocasionando uma postura inadequada. No estágio do $2^{\circ}$ trimestre, como o feto precisa de mais espaço para se desenvolver e acomodar-se, o músculo reto abdominal e os ligamentos afastam-se e aumenta ainda mais a instabilidade (Carvalho et al., 2017). E no $3^{\circ}$ trimestre ocorrem a alteração na postura e na forma de caminhar, consequentemente onde surgem mais desconfortos e dores na região lombar. Algumas mulheres limitam-se a execução de suas atividades diárias, devido ao peso da mama ou barriga, extensão das articulações e força muscular (Vieira \& Fleck, 2013).

Durante o período gestacional, a mulher passa por diversas mudanças. No sistema endócrino, com a liberação de hormônios devido a presença do feto, causando um aumento gradativo do volume uterino e abdominal. Ganho de peso na região abdominal gera uma sobrecarga ao assoalho pélvico, podendo desenvolver patologias como a incontinência urinária. Com o aumento do volume abdominal distende as fibras da pele fazendo com que inicie o aparecimento de estrias, diástase, mudança no padrão respiratório, desativação da musculatura, desconforto para andar, sentar-se, deitar-se, aparecimento de dores lombares e predisposição sexual. Essas mudanças, se não tratadas, podem interferir na qualidade de vida da gestante (Peruzzi \& Batista, 2018).

Órgãos nacionais e internacionais, e com os avanços tecnológicos e médicos, buscando proporcionar as gestantes segurança na gestação, incentivam elas a praticar exercícios físicos, visando a promoção da saúde, evitando a mortalidade materna e fetal. Os aspectos relacionados entre o trabalho de parto e a atividade física são: facilidade do trabalho de parto, redução do número de cesáreas, risco de parto prematuro e melhor recuperação pós-parto (Batista et al., 2003).

O fisioterapeuta é profissional adequado para atenuar as sintomatologias gestacional, trabalhando com medidas preventivas. Através da anamnese física, identifica-se as alterações pela avaliação postural, opinando sobre quais exercícios realizar, a intensidade e duração, podendo-se estender até pós-parto. Baseia-se por exercícios de alongamentos, respiração, relaxamento e fortalecimento dos músculos específicos e sobrecarregados na gestação, trabalhando também a musculatura do assoalho pélvico e abdominal, postura corporal, auxiliando na melhoria das lombalgias, queixas de câimbras, falta de ar e retenção, fazendo com que a gestante tenha uma melhor qualidade de vida e preparo para o parto, que são de estrema importância nesse período (Oliveira; Barros \& Araujo, 2010).

Além do preparo global que a fisioterapia interage, é de grande importância que toda gestante faça exercícios para fortalecimento do assoalho pélvico, que é uma rede músculo da cavidade inferior da pelve, que tem como importância sustentar a bexiga, útero, intestino, e controla a urina, fezes e funções sexuais. O fisioterapeuta elabora os exercícios de acordo com a 
necessidade de cada grávida, devendo ser específico, individualizado e exclusivo, visando melhoria na força, flexibilidade e condicionamento físico. Deve-se tomar cuidado com a exaustão ou fadiga. Os exercícios podem ser realizados no solo ou na piscina (hidroterapia), com indicação de início a partir do segundo trimestre de gravidez, quando os riscos de aborto em uma gravidez normal são pequenos (Coimbra; Souza \& Delfino, 2016).

O presente trabalho tem por objetivo compilar na literatura sobre a importância da atuação do fisioterapeuta na gestação. $\mathrm{O}$ estudo tem como finalidade apresentar a importância da fisioterapia no período gestacional, sendo que exercícios físicos praticados por gestantes podem colaborar de forma fundamental para a melhoria física e emocional da grávida, além do fato de que a gestante que realiza exercícios físicos regular podem evitar doenças decorrentes nesse período, minimizando os índices de obesidade e melhorando consideravelmente a qualidade de vida.

\section{Metodologia}

O estudo trata-se de uma revisão bibliográfica e descritiva de abordagem qualitativa. Tendo como base para sua discussão teórica, artigos científicos e demais produções intelectuais (Pereira et al., 2018).

A coleta de informações foi realizada em bibliotecas da área de saúde, revistas eletrônicas e bases de dados virtuais, como: Pubmed (National Library of Medicine), Medline (Medical Literature Analysis and Retrieval System Online) e Scielo (Scientific Eletronic Library Online). O trabalho tem como disposição os seguintes descritores: Fisioterapia gestacional; gestação; atuação do fisioterapeuta. Após a identificação dos textos, realizou-se uma leitura criteriosa a fim de selecionar os artigos que estão compondo essa revisão de literatura.

\section{Resultados e Discussão}

\subsection{Período gestacional}

A gestação é um período em que a mulher guarda por nove meses um ser que surgiu do encontro de células sexuais (espermatozoide e óvulo) no momento da cópula. Essa mulher vive um período com transformações únicas no seu corpo devido as alterações fisiológicas, biológico e psíquico próprias da gestação e essas repercussões variam de gestante para gestante e da idade gestacional. (Girard et al., 2019). As modificações sistêmicas são mais evidenciadas, pois se relacionam aos sistemas cardiovascular, respiratório, urinário, metabólico, gastrointestinal, urogenitais, biomecânicas, entre outros (Ferreira et al, 2017).

$\mathrm{Na}$ gestação, a mulher passa por modificações hormonais, mudanças decorrentes da interação e aumento de alguns hormônios, sendo progesterona, o estrogênio e a relaxina. Estes hormônios são responsáveis pelo fortalecimento e preparo dos músculos da região pélvica, e desenvolvimento do feto. Sintomas da gravidez são definidos como: vômitos, náuseas, vertigem, constipação, palpitações, insônia, cãibras, sonolência, varizes e lombalgia, relacionados a prejuízos domésticos e profissionais. Cada organismo reage diferente, significando que a mulher precisará adaptar-se e modificar sua rotina, e para melhorar as alterações fisiológicas é necessário incluir a prática de exercícios (Baracho, 2012).

As alterações fisiológicas e estruturais decorrentes da gravidez podem interferir tanto no bem-estar diário da gestante, quanto na hora do parto, refletindo diretamente na saúde do recém-nascido (RN). A lombalgia é uma das principais queixas das gestantes, que pode ser definida como dor e rigidez na região inferior do dorso. Esta dor se manifesta de várias maneiras, tais elas como: dor na coluna lombar, dor no quadril, impossibilitando suas habilidades físicas da vida diária, causadas pelo sobrepeso, má postura dentre outras alterações da gestação (Ferreira et al., 2017).

\subsection{Principais alterações no período gestacional}

No período gestacional a mulher passa por diversas alterações, tais como: crescimento uterino, além do peso do feto, reduz o espaço da bexiga, promovendo uma pressão sobre as estruturas do assoalho pélvico, onde vai ocasionar um 
enfraquecimento na região, devido a pressão gerada pela sobrecarga (Delancey, 2010). Segundo Coyne afirma que o corpo da gestante passa por processos fisiológicos de alterações hormonais, com diminuição da função muscular do assoalho pélvico, sendo um importante potencial para o aparecimento da incontinência urinária (IU), ou seja, em torno de 50\% das gestantes apresentam perda de urina no período gestacional. Foi definida pela International Continence Society (ICS) que a incontinência urinária é uma perda involuntário da urina, uma vez que a gravidez traz diversas alterações fisiológicas e anatômicas, sendo uma delas o aumento do útero (Coyne et al., 2012).

As alterações gestacionais podem ocasionar um quadro álgico significativo. Com o crescimento fetal, sobrecarrega a coluna lombar, modificando a postura da gestante e surgindo a lordose lombar. Mudanças hormonais, aumenta a produção do hormônio relaxina causando frouxidão nas articulações, que ocorre um desequilíbrio do sistema osteomioarticular. O aumento das mamas há um crescimento da cifose torácica, estiramento da musculatura do abdômen surge tensão nos músculos paravertebrais. Na reta final ocorre modificação nos ombros, provocando um aumento na curvatura da coluna a fim de adaptarse a postura e manter equilíbrio corporal (Kisner \& Colby, 2005).

Com as modificações uterinas e de acordo com o desenvolvimento fetal, o diafragma que é o músculo responsável pela respiração, altera sua posição, por ter pouco espaço, por isso as gestantes sentem dificuldades em respirar, principalmente ao realizar esforços. A frequência cardíaca aumenta nos primeiros meses e a pressão arterial diminui, e normaliza após o primeiro trimestre (Marques \& Sanches, 1994).

Figura 1. Incontinência urinaria

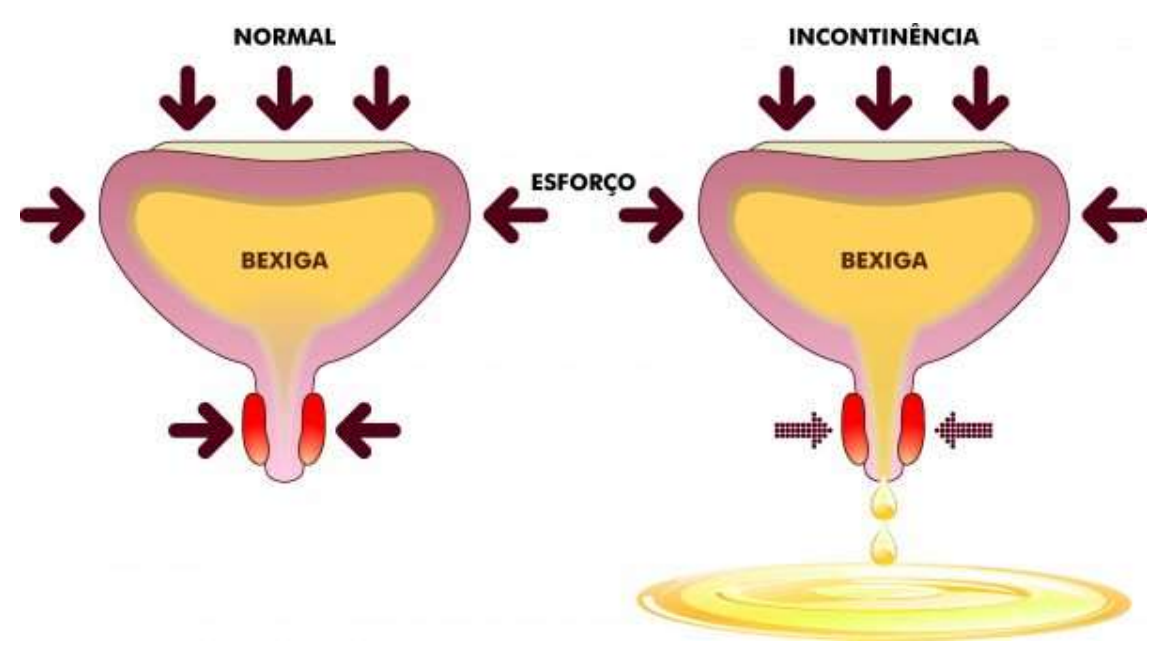

Fonte: http://incontinenciaurinariabcs.org/2017/05/31/incontinencia-urinaria-de-esforco/\#: :text=Multim\%C3\%ADdia-,I NCO NTIN\% C3\% 8ANCIA\%20URIN\%C3\%81RIA\%20DE\%20ESFOR\%C3\%87O,-Voltar\%20\%C3\%A0 \%20p \%C3\%A1gina

\subsection{O papel da fisioterapia no período gestacional}

A fisioterapia na obstetrícia, visa auxiliar a mulher a se adaptar às mudanças físicas do início ao fim da gestação e pósparto, minimizando o estresse. Realiza uma avaliação e tratamento de patologias como: o padrão respiratório, postural e na preparação para ajudar na hora do trabalho de parto. Tem como objetivo não apenas tratar, mas também prevenir com orientação na tentativa de uma intervenção precoce se possível. A fisioterapia age no papel de amenizar o sofrimento e restabelecer um padrão de saúde que está em desequilíbrio, restabelecer funções para facilitar as atividades de vida diária (Nobrega \& Barbosa, 2012).

O Fisioterapeuta além de aborda sobre as mudanças fisiológicas das gestantes, também atua no emocional, com o 
profissional de suporte à parturiente e usando múltiplos recursos para o controle da dor no trabalho de parto. Com sua assistência o profissional contribuirá para um ótimo trabalho de parto e o bem-estar da mãe e do bebê. Outra função é examinar e tratar gestantes com comprometimentos esqueléticos específicos compreendendo o conhecimento de lesão e regeneração dos tecidos. Realizar uma educação, talvez pela primeira vez as gestantes, sobre o papel dos músculos do assoalho pélvico (Souza \& Claúdia, 2014).

Um dos objetivos principais é a melhora da qualidade de vida da gestante. Por meio de avaliações individual é traçado um programa de exercícios e orientações sobre a realização das atividades diárias às quais o fisioterapeuta fará as adaptações às circunstâncias e realidade da gestante; é importante também um apoio psicológico durante esse período. Além de contribuir na autoestima, também evita o ganho excessivo de peso, sendo comum nesse período (Kisner \& Colby, 2005).

A Fisioterapia consiste no treinamento da musculatura do assoalho pélvico, visando um ganho de resistência, força e de controle de contração muscular, melhorando a sustentação do assoalho pélvico envolvendo grandes grupos musculares e atividades que desenvolvam a força e alongamento pois durante o parto é importante que os músculos possam ser alongados para facilitar a passagem do bebê no canal da vagina, evitando assim possíveis lesões musculares como laceração ou episiotomia (Mazzali \& Nascimento, 2008).

É precioso preparar o corpo para as alterações fisiológicas que são decorrentes da gestação, bem como fortalecer os músculos e melhorar condicionamento físico. Os principais exercícios que devem ser evitados são os de alto impacto. Orientase caminhadas, exercícios aeróbicos, preparando o corpo da gestante para o trabalho de parto, trazendo benefícios para o sistema cardiopulmonar e previne alterações posturais, entre outros (Stephenson; o'Connor, 2004).

A aprendizado de exercícios no período da gravidez é fundamental, pois melhora o condicionamento físico, ajuda no metabolismo, onde vai esta diminui edema, também trabalhar a respiração e condiciona o corpo para o trabalho de parto. Dentre os exercícios indicados para gestantes. (Rodrigues, Alves, Penna, Pereira, Branco \& Silva.2015).

A cinesioterapia e um recuso que se baseia no conceito de movimentos voluntários repetidos aonde vai está gerando força muscular. Tem como finalidade a mobilização do corpo do indivíduo. com algumas serias de exercícios onde vai trazer prevenção da rigidez muscular e a estimulação de um músculo, diminuindo contraturas, recuperando ou mantendo a força muscular, restituindo a função motora, nutrindo os tecidos e com todos esses fatores, vai estar preparado a mulher para o parto. (Xhardez, 2000).

A cinesioterapia do assoalho pélvico basear no princípio de que as contrações voluntárias acrescentam a força muscular, não possui contraindicação, e um método no qual pode ser realizando com os objetivos de aumenta a força, coordenação motora dos movimentos e da resistência muscular. (Silva, 2011).

Ponte - Paciente deitado em decúbito dorsal joelhos flexionados com os pés apoiados no chão, e eleva o quadril, ficando apoiada apenas sobre os ombros e os pés. Deve se realiza contração dos glúteos, contando até 3 segundos e depois vai relaxar essa musculatura. Repetindo estas contrações em três séries de 10 vezes (Baracho, 2007).

Kegel - São indicados para fortalecer o assoalho pélvico. Trata-se de um exercício seguro e eficaz que pode ser realizado antes, durante e depois da gravidez. Exercícios de kegel consistir em alongamentos globais, que trabalhar propriocepção da musculatura do assolho pélvico, pode se realizando da seguinte maneira. (Exercícios são realizando dentro dos limites de cada paciente) (Dias, Dias, Santos, Bitencourt, Oliveira \& Nunes 2016).

- A: Paciente em Decúbito dorsal, posiciona as pernas semi-fletidas, pés no chão, vai realizar uma expiração, posicionar a pelve em retroversão, elevar as nádegas ainda em retroversão. Repousar lentamente enquanto inspirava desenrolar lentamente a região lombar até o solo;

- B: Paciente em Decúbito dorsal, nádegas ligeiramente elevadas com uma almofada, pernas flexionadas e cruzadas, vai posicionar os pés no chão; sustentar entre as faces internas do joelho; elevar quadril o mais alto possível enquanto expira, voltar 
à posição de partida enquanto inspira lentamente;

- C: Paciente em Decúbito dorsal, nádegas apoiadas no chão, posiciona uma bola entre as pernas e vai realizar uma elevação das duas pernas semi-estendidas;

- D: Paciente em Decúbito dorsal, nádegas ligeiramente elevadas, vai apoia as pernas flexionadas e outra vai realizar uma elevação estendida. Realizar o exercício com as uma perna depois a outra;

- E: Paciente Em pé, com uma bola posicionadas entre as faces internas da coxa, ficar na ponta dos pés, contraindo o períneo e relaxando-o ao voltar com as plantas dos pés no chão;

- F: Paciente Sentada com as duas pernas estendidas vai realizar uma contração da musculatura perineal;

- G: Paciente Em pé, vai encostada em uma parede realizar retroversão da pelve com a musculatura pélvica contraída.

Mobilidade pélvica - consiste em movimentos que vai facilitar o encaixe do bebê, promovendo alívio das dores. São movimentos que podem ser feitos deitados sentados ou em pé. A gestante em pé, mãos apoiadas na cintura fazendo movimentos mobilizando a pelve de um lado para o outro como se estivesse dançando, posição de gato, paciente em pé joelho semiflexionados mãos apoiadas em corrimão ou até mesmo no próprio joelho. Os movimentos devem ser feitos durante os intervalos de contração. (Pereira; Mascarenhas \& Gramacho, 2016).

Bola Suíça - é um recurso utilizado durante o trabalho de parto que vai auxiliar em movimentos pélvico, onde vai trazer relaxamento perineal, diminuindo a dor e facilitando a descida do feto é vai está promovendo um fortalecimento dos músculo levantador do ânus e o pubo coccígeo (Oliveira et al., 2014). Além de estar promovendo a mulher, a ter um maior conforto e possibilitado uma posições adequadas durante o processo do parto (Santana et al., 2016).

Massagem - é um recurso que auxilia no alívio de dor, relaxamento muscular, nos desconfortos reduzindo o estresse, ansiedade, reduzindo a fadiga, promovendo efeito sedativo, aumento da consciência corpórea, melhora do equilíbrio entre o sistema simpático e parassimpático. Apesar de ser pouco os consentimentos dessas técnicas, as gestantes relatam que a técnica traz tranquilidade, reduz a ansiedade e traz analgesia. Massagem pode ser feita de forma suave de um lado para o outro, e amassamento, em toda região lombar e sacral (Bavaresco et al., 2011).

Reflexologia - é uma técnica oriental que promove o alívio da dor. Consiste em áreas de reflexos das nossas mãos e pés que refletem uma imagem de todo o nosso corpo precisamente mesma ordem e posição que no corpo. Existe diferentes interpretações quanto ao mecanismo de ação da reflexologia, algumas teorias foram propostas, tais quais: teoria do controle de portão da dor e a teoria do impulso neural, que aumenta a secreção de endorfinas e encefálicas que auxiliam no alívio a dor, e no desenvolvimento do fluxo sanguíneo e linfático, contribuindo para eliminação de toxinas do corpo (Dolatian et al., 2011).

\subsection{Exercícios respiratórios}

Os treinamentos respiratórios tem como papel no trabalho de parto reduzir a sensação dolorosa, e otimizar os níveis de saturação materno-fetal, reduzir os níveis de tensão e garantir um melhor relaxamento (Gallo et al., 2011). Exercícios respiratório são importantes durante o trabalho de parto, pois ajuda no relaxamento, e promove concentração, diminuído os riscos de possíveis traumas perineais no período expulsivo. Também melhora a oxigenação da mãe e do feto. No momento expulsivo, a parturiente muitas vezes apresenta-se muito agitada, realizando respirações curtas e ofegantes durante os intervalos das contrações (Coimbra, 2016). O fisioterapeuta vai está orientando a parturiente a esta realizar uma respiração profunda, enchendo todo a cavidade do pulmão e a expiração é feita lentamente com os lábios franzidos no momento de força para a expulsão do bebê (Nunes; Moreira \& Vial, 2015). 


\section{Conclusão}

Sabemos que o período gestacional traz muitas alterações fisiológicas, desconfortos e dores para as futuras mamães. Uma forma de prevenir ou minimizar é com a prática de exercícios, uma gestante ativa tem muitos benefícios em relação às sedentárias, inclusive com um parto e um pós-parto mais tranquilos.

Através dos resultados obtidos no presente estudo, o fisioterapeuta tem ação importante tanto no período gestacional quanto no período de pós-parto, possui técnicas que melhora a dor, a postura corporal, contribui no parto, minimizando lesões no assoalho pélvico. O fisioterapeuta é responsável por fazer a anamnese, orientar as pacientes sobre como é o período gestacional, as alterações fisiológicas, as possíveis patologias, os tipos de parto, os exercícios realizados, e os acontecimentos pós-parto. Vale ressaltar que através da literatura observa-se poucas divulgações de artigos na área, como a falta de conhecimento da atuação do fisioterapeuta, dos tipos de exercícios realizados, a falta de conhecimento também por parte das gestantes, chegando à conclusão que é importante expandir e divulgar a fisioterapia, seus procedimentos e benefícios. Falta a integração do fisioterapeuta em todas as equipes multidisciplinar, atuando diretamente no planejamento familiar, para realizar o acompanhamento obstetrício, seja para tratamento ou prevenção de alguma patologia.

Espera-se que os conhecimentos relatados por este estudo possam aumentar a visibilidade da fisioterapia na gravidez, viabilizando uma maior procura por essa assistência prestada as gestantes, no pré-natal e após o parto, trazendo assim os benefícios proporcionados por essa prática.

Através desse trabalho foi notado a relevância de falar mais sobre a fisioterapia no período gestacional, em trabalhos futuros poderemos aprimorar mais sobre esse assunto que e tão rico em informações e pouco discutindo entre as gestantes, abordado especificamente os principais cuidados e prevenções que deve se ter durante cada fase da gestação.

\section{Referências}

Baracho, E. (2012). Fisioterapia aplicada à saúde da mulher. (5a ed.), Guanabara Koogan.

Batista, D., Chiara, V., Gugelmin, Â., \& Martins, P. (2003). A atividade física e gestação: saúde da gestante não atleta e crescimento fetal. Revista Brasileira Saúde Materno Infantil. 3. 151-8.

Bavaresco, G. Z. et al. (2011) O Fisioterapeuta como Profissional de Suporte à Parturiente. Ciência \& Saúde Coletiva, ,16, (7), .3259- 3266.

Baracho \& Elza. (2007). Fisioterapia aplicada a obstetrícia, uroginecologia e aspectos de mastologia. Guanabara Koogan.

Carvalho, M. E. C. C., et al. Lombalgia na gestação. (2017) Revista Brasileira de Anestesiologia. 266-270. 63a ed.

Coimbra, F. R., S. Bruna C. d. D., Marta M. (2016) Fisioterapia no suporte a parturientes. Revista Científica da FEPI-Revista Científica Universitas.

Coyne, B. et al. (2012). Urinary incontinence and its relationship to mental health and health-related quality of life in men and women in Sweden, the United Kingdom, and the United States. Rev Eur Urol, 61, (1), 88-95.

Delancey, J. (2010). Why do women have stress urinary incontinence. Neurourol Urodyn, .1, (.29).7-13.

Dolatian, M. et al 2011. O efeito da reflexologia na intensidade da dor e durante o parto em primíparas. Iranian Red Crescent Medical Journal, Dubai. 13, (7), 475-479.

Gallo, R. B. S., Santana, L. S., Marcolin, A. C., Ferreira, C. H. J., Duarte, G. \& Quintana, S. M. (2011). Recursos não farmacológicos no trabalho de parto: protocolo assistencial. Femina, São Paulo.39, (1), 41-8.

ABC. (2017). Incontinência uninária de esforço. Website da Associação Brasileira pela Continência (ABC). Incontinenciaurinariabcs.o rg/2017/05/31/inconti nencia-urinaria-de-esforco/\#: :text=Multim\%C3\%ADdia-, Incontin\%C3\%8ANCIA\%20URIN\%C3\%81RIA\%2 0DE\%20ESFOR\%C3\% 87O,Voltar\%20\%C3\%A0\%20p\%C3\%A1gina.

Kisner, C. \& Colby, L. A. (2005). Exercícios terapêuticos fundamentos e técnicas. (4a ed.),

Marques, A. P., Sanches, E. L. (1994). Origem e evolução da fisioterapia: aspectos históricos e legais. Revista de fisioterapia. Universidade de São Paulo, 1(1): $5-10$.

MazzalI, L., Nascimento G. \& Ronald (2008). Análise do Tratamento Fisioterapêutico na Diminuição da Dor Durante o Trabalho de Parto Normal. - Ensaio e Ciências: C. Biológicas, Agrárias e de Saúde. 12. 
Research, Society and Development, v. 10, n. 11, e480101119977, 2021

(CC BY 4.0) | ISSN 2525-3409 | DOI: http://dx.doi.org/10.33448/rsd-v10i11.19977

Nóbrega, M. T. Leite, A. C.; \& Barbosa, C. A. K. K. (2012). Diástase dos retos abdominais em puérperas e sua relação com variáveis obstétricas. Fisiot. Mov.25(2).

Nunes, G. da S., Moreira, P. C. de S. \& Vial, D. de S. (2015). Recursos fisioterapêuticos para o alívio da dor no trabalho de parto. Revista FAIPE, 5(1), 90-99.

Oliveira, C. F. P., Barros, D. J. M. \& Araujo, F. A. B (2010). A incidência de dores musculoesqueléticas na gestação. Monografia (Graduação em fisioterapia) - Unisalesiano, Lins.

Oliveira, L. M. N. \& Cruz, A. G. C. (2014). A Utilização da Bola Suíça na Promoção do Parto Humanizado. Revista Brasileira de Ciências da Saúde, São Caetano do Sul.18 (2),175-180.

Pereira A. S. et al. (2018). Metodologia da pesquisa científica. UFSM

Pereira, T. C. B., Mascarenhas, T. R. \& Gramacho, R. C. C. V. (2016). Métodos não farmacológicos para alívio da dor no trabalho de parto: uma revisão sistemática de literatura.

Peruzzi, J.; \& Batista, P. A. Fisioterapia nas disfunções do assoalho pélvico e na sexualidade durante o período gestacional, Revista Fisioterapia Brasil, 19(2):177-182.

Rodrigues, D. P., Alves, V. H., Penna, L. H. G., Pereira, A. V., Branco, M. B. L. R., \& Silvia. L. A Violência no campo obstétrico, Escola Anna Nery 19(4)

Stephenson, R. G. \& O'Connor, L. J. (2004). Fisioterapia aplicada à ginecologia e obstetrícia. (2a ed.), Manole, 12-20,177-194.

Souza S. E. R. \& Cláudia O. (2014). Influência da Cinesioterapia na fase ativa do trabalho de parto no centro de pré-parto, parto e pós-parto do Instituto da Mulher dona Lindu - Faculdade Ávila.

Santana, L. S. Gallo, R. B. S., Ferreira, C. H. J., Quintana, S. M \& Marcolin, A. C. (2016). Transcutaneous electrical nerve stimulation (TENS) reduces pain and postpones the need for pharmacological analgesia during labour: a randomised trial. Journal of Physiotherapy, 62 .29-34.

Silva, C. R. (2011). Cinesioterapia do Assoalho Pélvico: abordagem fisioterapêutica na incontinência urinária e nas disfunções sexuais femininas. Phorte.

Vieira, T. M. da C., \& Fleck, C. S. 2013 A influência do método pilates na dor Lombar crônica: uma revisão integrativa. (2a ed.) Disciplinarum Scientia. 14, 285-292.

Xhardez, Y. (2000). Manual de Cinésioterapia. Atheneu.

Dias, E., Dias, J. M., Santos, L. M., Bitencourt, A. C. P., Oliveira, N. K. S., \& Nunes, G. G. F. (2016). Cinesioterapia como tratamento da incontinência urinária de esforço - estudo de caso. Rev Eletr ES. 5 (1): 61-72. 